\title{
INHIBITION OF YEAST MITOCHONDRIAL NUCLEOID FUSION BY ETHIDIUM BROMIDE AND RESPIRATION INHIBITORS
}

\author{
ISAMU MIYAKAWA, TETSUJI TSUKAMOTO, MASAMI SAKODA, \\ TSUNEYOSHI KUROIWA, * AND NOBUNDO SANDO \\ Biological Institute, Faculty of Science, Yamaguchi University, \\ Yamaguchi 753, Japan \\ * Department of Biology, Hongo, Faculty of Science, University of Tokyo, \\ Tokyo 113, Japan
}

(Received November 21, 1988)

\begin{abstract}
Yeast spheroplasts were cultured in several sporulation media, each containing different kinds of inhibitors which affected meiosis, and the morphology of the mitochondrial nucleoids (mt-nucleoids) was examined using the DNA-binding fluorescent dye, 4',6-diamidino-2-phenylindole. Adding inhibitors which completely blocked meiosis and sporulation caused the mt-nucleoids to form two distinct morphological types. In the presence of actinomycin $\mathrm{D}$, cycloheximide, methyl 2-benzimidazole carbamate and 2-(4-thiazolyl)-1 $\mathrm{H}$-benzimidazole, the mt-nucleoids fused with each other and showed a marked string-like appearance, as did the mtnucleoids observed in normal sporulation process. In the presence of ethidium bromide, antimycin A, olygomycin, carbonyl cyanide $\mathrm{m}$ chlorophenylhydrazone, mt-nucleoids appeared as fluorescent particles scattered in the cytoplasm. However, adding glucose to the sporulation medium containing antimycin A gave rise to a marked string-like appearance of mt-nucleoids in spite of strong inhibition of respiration. These results indicated that the morphology of mt-nucleoids was not directly associated with respiration itself, but that the preservation of complete mitochondrial DNAs and some metabolic activities related to respiration were required for the formation of string-like mt-nucleoids in sporulation media.
\end{abstract}

Recombination and segregation of mitochondrial genomes have been extensively studied in Saccharomyces cerevisiae as a representative of cytoplasmic inheritance in eucaryote(l). In contrast with these genetic studies, cytological

\footnotetext{
Address reprint requests to: Dr. Isamu Miyakawa, Biological Institute, Faculty of Science, Yamaguchi University, Yamaguchi 753, Japan.
} 
studies of mitochondrial genomes have been so limited that very little structural evidence for various genetic occurrences among them has been presented.

For the last two decades, a yeast mitochondrial nucleoid (mt-nucleoid) has been defined in electron microscopy as a region that is distinguished from other regions of the mitochondrial matrix by the appearance of DNA fibres and low electron density (2). However, fluorescence microscopy, using 4',6-diamidino-2phenylindole (DAPI) (3), is an easier and more effective method for detecting yeast mt-nucleoids, since it has higher resolution(2-6). By using DAPI-fluorescence microscopy we have shown previously that mt-nucleoids, which were scattered as fluorescent spherules in the cytoplasm of stationary phase cells, formed a string-like network in a branched giant mitochondrion during the sporulation process $(4,5)$. Thus, the sporulation culture using spheroplasts is appropriate for investigating the behavior of mt-nucleoids because rapid morphological changes among the mtnucleoids are easily induced by the transfer of spheroplasts into the sporulation medium, and the DAPI-stained mt-nucleoids in spheroplasts can be observed more clearly than in whole cells.

Since we believe that string-like mt-nucleoids, which appear as a result of mtnucleoid fusion in fluorescence microscopy, play an important role in recombination of mtDNAs (5), we were interested in the question of how morphological changes among mt-nucleoids were induced and what kinds of intracellular structures were associated with those changes. In this study, we investigated the behavior of mt-nucleoids in spheroplasts transferred to sporulation media containing several kinds of inhibitors in order to reveal the mechanism underlying the morphological change in mt-nucleoids.

\section{MATERIALS AND METHODS}

Strains and culture. Saccharomyces cerevisiae diploid strain G2-2, obtained by crossing strain 24428 (mating type $a$ ) with strain 3626 (mating type $\alpha$ ), was aerobically cultured in a modified Burkholder's medium (BT medium) under reciprocal shaking at $30^{\circ} \mathrm{C}(4)$. Fractionation of large cells, spheroplast formation, and the sporulation culture of spheroplasts were the same as we described previously $(4,5)$. The sporulation medium contained $15 \mathrm{~mm}$ potassium acetate, $50 \mathrm{~mm}$ potassium phosphate buffer $(\mathrm{pH} \mathrm{6.9)}$ and $0.6 \mathrm{M}$ sorbitol.

Addition of inhibitors. Each inhibitor was added to the sporulation cultures at time zero. Actinomycin D, cycloheximide and ethidium bromide were added at concentrations of $16,10,127 \mu \mathrm{M}$ from the stock solutions dissolved in water. Methyl 2-benzimidazole carbamate (MBC) and 2-(4-thiazolyl)-1H-benzimidazole (TBZ) were added at respective concentrations of 1.3 and $2.6 \mathrm{~mm}$ from 130 and $260 \mathrm{~mm}$ stock solutions dissolved in dimethyl sulfoxide. Antimycin A, oligomycin and carbonyl cyanide $m$-chlorophenylhydrazone (CCCP) were added at respective concentrations of $0.73 \mu \mathrm{M}, 2 \mu \mathrm{g} / \mathrm{ml}$ and $5.0 \mu \mathrm{M}$ from $365 \mu \mathrm{M}, 5 \mathrm{mg} / \mathrm{ml}, 2.5 \mathrm{~mm}$ stock solutions dissolved in ethanol. Chloramphenicol was added to the sporulation 
medium at a concentration of $5 \mathrm{mg} / \mathrm{ml}$.

Fluorescence microscopy. All observations were made with an Olympus BHSRFK epifluorescence microscope. Spheroplasts were fixed with $1 \%$ glutaraldehyde and stained with DAPI $(1 \mu \mathrm{g} / \mathrm{ml})$ as described previously (5).

Respiration activity. Portions of the sporulation cultures were sampled at regular intervals and the oxygen uptake was determined polarographically at $30^{\circ} \mathrm{C}$.

Determination of glucose concentration. The glucose concentration of the sporulation medium was determined by the DNPA method(7).

Isolation of rho ${ }^{-}$strain. Strain G2-2 was cultured for $1 \mathrm{~h}$ in a BT medium containing ethidium bromide $(50 \mu \mathrm{g} / \mathrm{ml})$. Then the cells were plated on BT-agar medium and the resulting small colonies were selected and tested with 2,3,5triphenyltetrazolium chloride (TTC) $(8)$ which stains respiratory-sufficient $\left(\right.$ rho $^{+}$) colonies red, while respiratory-deficient $\left(\mathrm{rho}^{-}\right)$colonies are not stained.

\section{RESULTS}

As shown in Fig. 1A, mt-nucleoids in the stationary phase spheroplasts were observed as 50-70 small fluorescent particles scattered throughout the cytoplasm. In meiosis, mt-nucleoids aligned with each other and formed a string-like network in the spheroplasts cultured for $6 \mathrm{~h}$ in the sporulation medium (Fig. 1B). In the first meiotic division, mt-nucleoids with a string-like appearance occurred near the dividing nucleus, and then a ring of mt-nucleoids enclosed each daughter nucleus after the second meiotic division $(4,5)$. The final sporulation rate was usually over $80 \%$.

However, in the presence of the inhibitor (Fig. 1), the cell nuclei remained single in all cells throughout the culture period, and chromatin condensation characteristic of meiosis $(5,9,10)$ was not found at all. The result indicated complete inhibition of meiosis.

In these cultures, the mt-nucleoids had two distinct types of morphology, depending on the kinds of inhibitors added. In the presence of actinomycin D, an inhibitor of RNA synthesis (Fig. 1C), and cycloheximide, an inhibitor of protein synthesis in cytoplasm (Fig. 1D), neighboring mt-nucleoids began to align just after initiation of the culture, and string-like mt-nucleoids similar to those seen in normally sporulating spheroplasts formed after $6 \mathrm{~h}$. In both cases, string-like mtnucleoids were maintained during the $24 \mathrm{~h}$ culture.

The string-like network of mt-nucleoids also appeared in spheroplasts cultured in the presence of the anti-microtubule drugs, MBC (Fig. 1E) and TBZ.

On the other hand, in the presence of ethidium bromide, which is the specific inhibitor of mitochondrial DNA and RNA syntheses, and causes extensive deletion of mitochondrial DNA (mtDNA), mt-nucleoids appeared as approximately 40 small spherules scattered in the reddish cytoplasm of spheroplasts cultured for $6 \mathrm{~h}$ (Fig. 1F). Then the number of mt-nucleoids gradually decreased, and after $24 \mathrm{~h}$, about 20 mt-nucleoids, which had an irregular size, were localized in part of the 

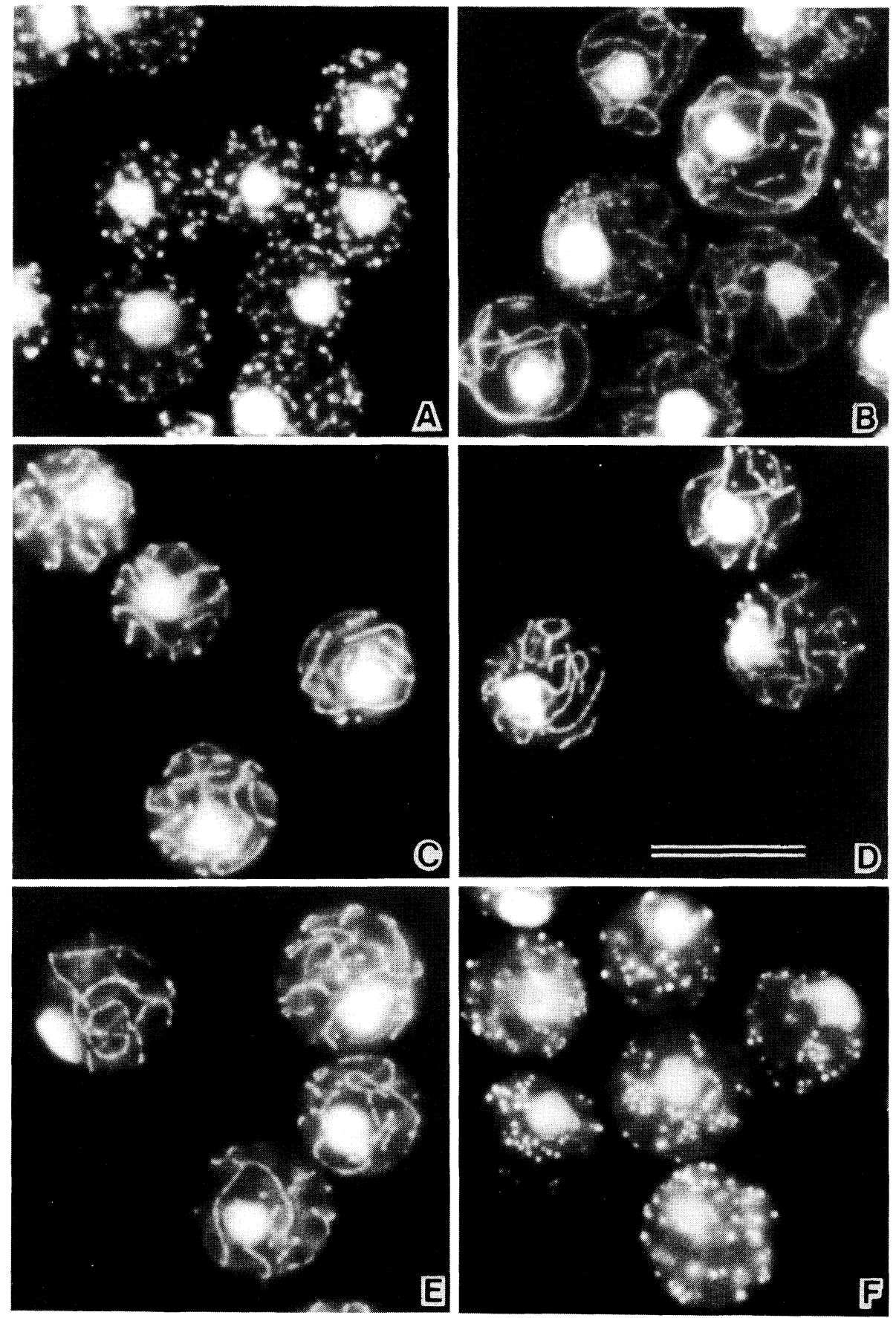

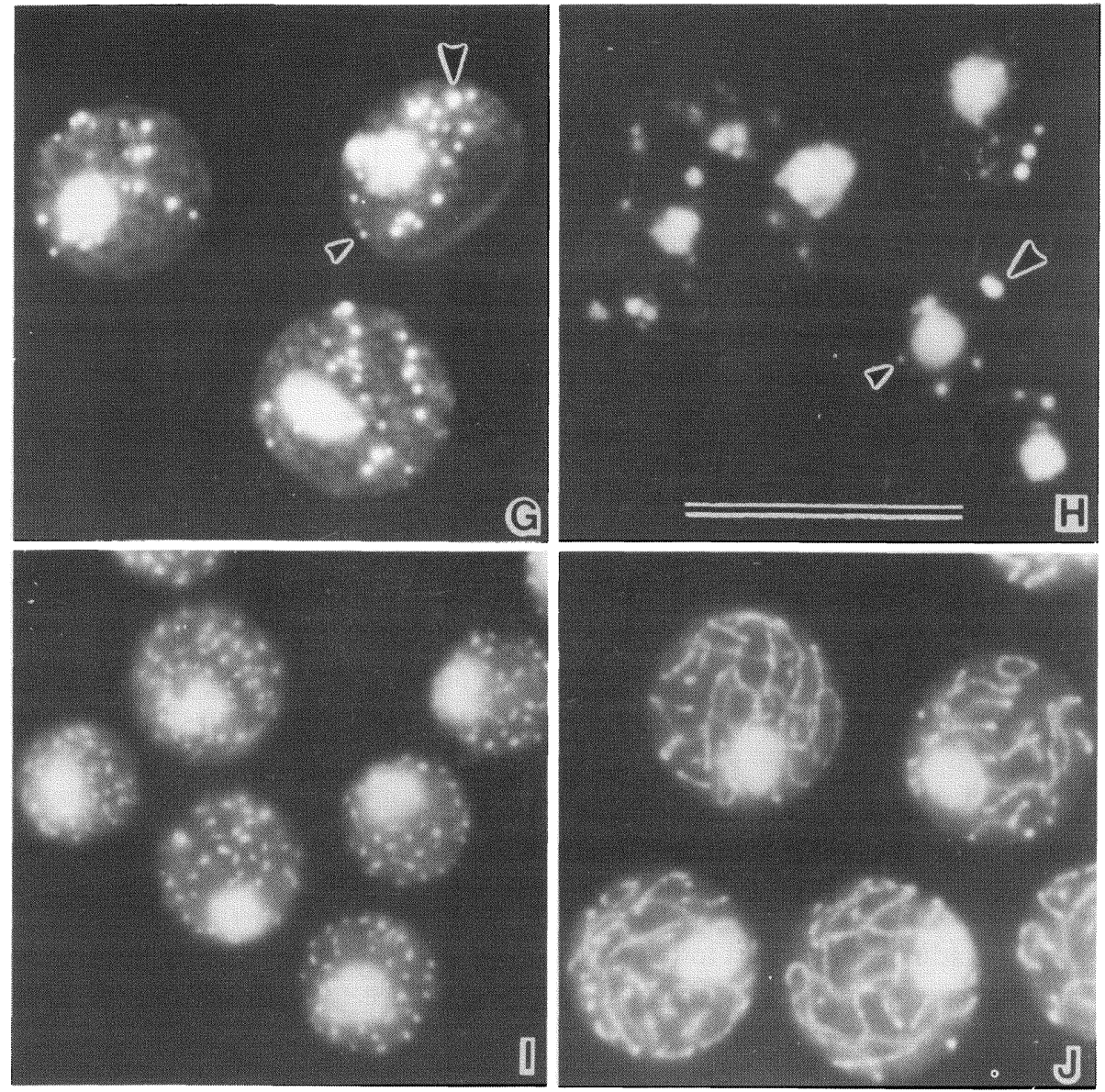

Fig. 1. Fluorescence micrographs showing cell nuclei and mt-nucleoids in spheroplasts after DAPI staining. Spheroplasts at stationary phase (A). Spheroplasts cultured for $6 \mathrm{~h}$ in sporulation medium (B). Spheroplasts cultured for $6 \mathrm{~h}$ in sporulation medium containing $16 \mu \mathrm{M}$ actinomycin D (C), $10 \mu \mathrm{M}$ cycloheximide (D), $1.3 \mathrm{mM} \mathrm{MBC}$ (E), $127 \mu \mathrm{M}$ ethidium bromide $(\mathrm{F})$. Spheroplasts cultured for $24 \mathrm{~h}$ in sporulation medium containing $127 \mu \mathrm{M}$ ethidium bromide (G). Rho ${ }^{-}$spheroplasts at stationary phase $(\mathrm{H})$. Spheroplasts cultured for $6 \mathrm{~h}$ in sporulation medium containing $0.73 \mu \mathrm{M}$ antimycin A (I). Three hcultured spheroplasts in sporulation medium containing both $0.73 \mu \mathrm{M}$ antimycin $\mathrm{A}$ and $7 \mathrm{~mm}$ glucose (J). In Fig. G, H, different sizes of mt-nucleoids are indicated by arrowheads. A-J except $\mathrm{H}$ are the same scale. Bar, $10 \mu \mathrm{m}$.

cytoplasm (Fig. 1G shown by arrowheads). About $10 \mathrm{mt}$-nucleoids, fewer than those in $\mathrm{rho}^{+}$cells, with irregular sizes (shown by arrowheads) also appeared in rho $^{-}$cells induced by treatment with ethidium bromide (Fig. $1 \mathrm{H}$ ).

In the presence of antimycin $\mathrm{A}$, an inhibitor of electron transport in mitochon- 


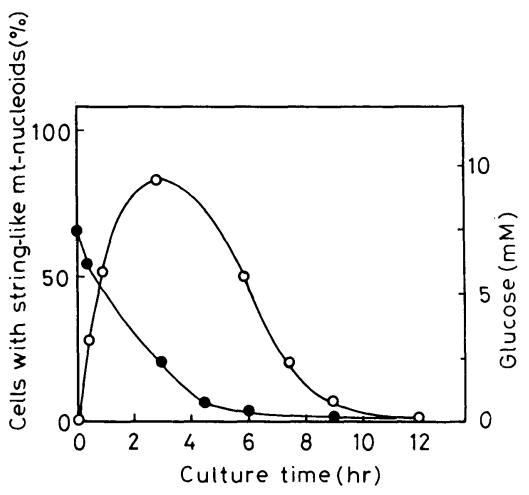

Fig. 2. Changes in morphology of mt-nucleoids in spheroplasts and glucose concentration during sporulation culture. The sporulation medium contained $0.73 \mu \mathrm{m}$ antimycin $\mathrm{A}$ and $7 \mathrm{~mm}$ glucose. $O$, percentage of spheroplasts which had string-like $\mathrm{mt}-$ nucleoids; $\bullet$, concentration of glucose in sporulation medium.

dria, the mt-nucleoids in almost all of the spheroplasts remained as small fluorescent particles, just like those in the spheroplasts at time zero, throughout the $24 \mathrm{~h}$ of the culture (Fig. 1I). The same effect on mt-nucleoids also occurred in oligomycin, an inhibitor of oxidative phosphorylation and CCCP, an uncoupler of mitochondria.

However, the addition of $7 \mathrm{~mm}$ glucose to the sporulation medium containing antimycin A caused a marked string-like appearance of mt-nucleoids (Fig. 1J, Fig. 2). The percentage of cells with string-like mt-nucleoids was more than $80 \%$ after $3 \mathrm{~h}$ and then gradually decreased to the level at time zero after $12 \mathrm{~h}$. The oxygen uptake of spheroplasts cultured for $0 \mathrm{~h}$ or $3 \mathrm{~h}$ in the presence of both antimycin $\mathrm{A}$ and glucose was strongly inhibited and was below $0.1 \times 10^{-7} \mu \mathrm{mol} / \mathrm{cell} / \mathrm{min}$, at both times. The dispersion of mt-nucleoids into small particles after $3 \mathrm{~h}$ apparently was related to the consumption of glucose in the culture medium (Fig. 2). On the other hand, the percentage of cells with string-like mt-nucleoids in normal sporulation medium increased with the progress of meiosis, and reached approximately $25 \%$ after $3 \mathrm{~h}, 50 \%$ after $6 \mathrm{~h}, 90 \%$ after $10 \mathrm{~h}$. Mature asci appeared after $12 \mathrm{~h}$. In the sporulation medium containing $7 \mathrm{~mm}$ glucose, the appearance of mature asci was delayed about $3 \mathrm{~h}$, and the percentage of cells with string-like mt-nucleoids was somewhat lower than that in normal sporulation medium. The oxygen uptake of normally sporulating spheroplasts cultured for $0 \mathrm{~h}$ or $3 \mathrm{~h}$ was 1.09 and $3.18 \times 10^{-7}$ $\mu \mathrm{mol} / \mathrm{cell} / \mathrm{min}$ respectively. These results indicated that marked string-like mtnucleoids were formed independently of meiosis, despite strong inhibition of oxygen uptake in the presence of both antimycin $\mathrm{A}$ and glucose.

In the presence of chloramphenicol $(5 \mathrm{mg} / \mathrm{ml})$, an inhibitor of protein synthesis in mitochondria, sporulation was only partially inhibited (about $50 \%$ sporulation) and string-like mt-nucleoids occurred in both sporulating and non-sporulating spheroplasts. However, observation of DAPI-stained mt-nucleoids became difficult 
after $15 \mathrm{~s}$ due to many yellowish particles which appeared soon after exposure to ultraviolet light.

\section{DISCUSSION}

Biogenesis of mitochondria depends on the cooperative expression of nuclear and mitochondrial genes $(I)$. Therefore, it was first supposed that some nuclear gene products expressed during meiosis participated in the string formation of $\mathrm{mt}-$ nucleoids. However, the results suggest that morphological change of mt-nucleoids did not need newly synthesized RNAs and proteins in cytoplasm during meiosis, since actinomycin $\mathrm{D}$ and cycloheximide did not inhibit the string formation of $\mathrm{mt}-$ nucleoids. The possibility that the participation of mitochondrial genes in the morphological change of mt-nucleoids was considered. But in the presence of chloramphenicol in high concentration, the inhibition of meiosis was incomplete, and string-like mt-nucleoids were also observed in these spheroplasts. The participation of mitochondrial genes on the morphology of mt-nucleoids remains ambiguous at the present time.

Furthermore, the active state of respiration in mitochondria was assumed to be associated with the string-like network of mt-nucleoids, as the participation of functional mitochondria is necessary for sporulation (11). The effect of respiration inhibitors, which completely blocked the formation of string-like mt-nucleoids in the sporulation medium, was consistent with this assumption. However, the results shown in Fig. 2 indicate that the morphology of mt-nucleoids was not directly associated with respiration itself, since string-like mt-nucleoids were formed despite strong inhibition of oxygen uptake by the addition of glucose to the sporulation medium containing antimycin A. It is probable that some other metabolic activities related to respiration in mitochondria or in cells participate in the formation of $\mathrm{mt}-$ nucleoid network.

The presence of a smaller number of mt-nucleoids that tended to aggregate was a unique feature of the spheroplasts treated with ethidium bromide and rho ${ }^{-}$cells. The morphological change among mt-nucleoids in the presence of ethidium bromide represents the process of production of mt-nucleoids that had a deletion in mtDNAs. Preservation of complete mitochondrial DNAs is required for the formation of string-like mt-nucleoids. Electron-microscopically the mitochondria in rho $^{-}$cells show distinct morphological alterations, especially in cristae(2). The distribution of mt-nucleoids in rho ${ }^{-}$cells may be intimately related with an aberrant form of mitochondria.

There have been many reports revealing the intimate association of microtubules with mitochondria in many organisms(12-16). So anti-microtubule drugs were expected to affect the behavior of mt-nucleoids. However, our study of the effects of MBC and TBZ did not support an active participation of cytoplasmic microtubules in the morphological change of mt-nucleoids. As a preliminary examination, we carried out indirect immunofluorescence staining of the sphero- 
plasts with an anti-tubulin antibody, together with DAPI staining. In stationary phase spheroplasts, several cytoplasmic microtubules emanated from the single spindle pole body (SPB) toward the cytoplasm. At meiotic prophase, the cytoplasmic microtubules disappeared and bundles of microtubules elongated from a single SPB into the nucleus. No cytoplasmic microtubules having an intimate relation with mt-nucleoids were found in either stage (data not shown). We have previously shown by vital staining of mitochondria that a string formation of mt-nucleoids was accompanied with the fusion of mitochondria $(4,5)$. Higher resolution in immunofluorescence microscopy, which permits the detection of single microtubules, is needed to elucidate a spatial relation of cytoplasmic microtubules with mitochondria and mt-nucleoids.

We thank N. Araki, M. Furutani and K. Mori for their helpful advice and discussion during the present work. This work was supported by a Grant-in-Aid No. 63110001 from the Ministry of Education, Science, and Culture of Japan.

\section{REFERENCES}

1) I. H. Evans, In Yeast Genetics. Fundamental and Applied Aspects, ed. by J. F. T. Spencer, D. M. Spencer, and A. R. W. Smith, Springer-Verlag, New York (1983), p. 269.

2) B. J. Stevens, In The Molecular Biology of the Yeast Saccharomyces. Life Cycle and Inheritance, ed. by J. N. Strathern, E. W. Jones, and J. R. Broach, Cold Spring Harbor Laboratory, New York (1981), p. 471.

3) D. H. Williamson and D. J. Fennell, In Methods in Cell Biology, Vol. 12, ed. by D. M. Prescott, Academic Press, New York (1975), p. 335.

4) N. Sando, I. Miyakawa, S. Nishibayashi, and T. Kuroiwa, J. Gen. Appl. Microbiol., 27, 511 (1981).

5) I. Miyakawa, H. Aoi, N. Sando, and T. Kuroiwa, J. Cell Sci., 66, 21 (1984).

6) I. Miyakawa, N. Sando, S. Kawano, S. Nakamura, and T. Kurolwa, J. Cell Sci., 88, 431 (1987).

7) T. Momose, A. Inaba, Y. Murai, and M. Watanabe, Talanta, 4, 33 (1960).

8) S. Nagai, N. Yanagishima, and H. Nagai, Bacteriol. Rev., 25, 404 (1961).

9) T. Kuroiwa, H. Kojima, I. Miyakawa, and N. Sando, Exp. Cell Res., 153, 259 (1984).

10) T. Kuroiwa, S. Miyamura, S. Kawano, M. Hizume, A. Toh-E, I. Miyakawa, and N. Sando, Exp. Cell Res., 165, 199 (1986).

11) B. Ephrussi, Nucleo-cytoplasmic Relations in Microorganisms, Clarendon Press, Oxford (1953).

12) M. H. Heggeness, M. I. Simon, and S. J. Singer, Proc. Natl. Acad. Sci. U.S.A., 75, 3863 (1978).

13) E. H. Ball and S. J. Singer, Proc. Natl. Acad. Sci. U.S.A., 79, 123 (1982).

14) Y. NaKamura and K. Ueda, Cytologia, 47, 713 (1982).

15) H. C. Hoch and R. C. Staples, Mycologia, 75, 795 (1983).

16) I. C. Summerhayes, D. Wong, and L. B. Chen, J. Cell Sci., 61, 87 (1983). 